Global behavior of solutions to a second order delay differential equation

\author{
H. Khatibzadeh
}




\title{
GLOBAL BEHAVIOR OF SOLUTIONS TO A SECOND ORDER DELAY DIFFERENTIAL EQUATION
}

\author{
H. KHATIBZADEH
}

Received 4 February, 2013

Abstract. In this paper, we study the asymptotic behavior of eventually positive (negative) and oscillatory solutions to the following nonhomogeneous second order delay differential equation

where $p$ and $f$ are suitable real functions.

$$
\left\{\begin{array}{l}
u^{\prime \prime}(t)=p(t) u(t-\tau)+f(t), \\
u(t)=\phi(t), \quad t \in[0, \tau),
\end{array}\right.
$$

2010 Mathematics Subject Classification: 39A23; 47H05

Keywords: delay differential equation, asymptotic behavior, asymptotic stability, bounded solution

\section{INTRODUCTION}

Qualitative theory of delay differential, difference and functional equations is the subject of many recent publications. Specially, asymptotic behavior and oscillation of delay differential and difference equations of first and second order has been studied by many authors. We refer the reader to the interesting book by Gopalsamy [1] and to the recent articles [2-5]. In this paper, we consider the following second order delay differential equation

$$
\left\{\begin{array}{l}
u^{\prime \prime}(t)=p(t) u(t-\tau)+f(t), \quad t \geq \tau \\
u(t)=\phi(t), \quad t \in[0, \tau)
\end{array}\right.
$$

where $\tau>0$ is a constant delay, and $p:[\tau,+\infty) \rightarrow \mathbb{R}^{+}, f:[\tau,+\infty) \rightarrow \mathbb{R}$, and $\phi:[0, \tau) \rightarrow \mathbb{R}$. In the special case $f(t) \equiv 0$ and $p(t) \equiv p>0$, the equation (1.1) has the characteristic equation $\lambda^{2}-p e^{-\lambda \tau}=0$. This equation has negative roots for suitable constants $p$ and $\tau$. For example, if $\tau=10$ and $p=\frac{1}{1000}$, then the equation $g(\lambda)=1000 \lambda^{2}-e^{-10 \lambda}$ has two negative roots $-\frac{1}{10}<\lambda_{1}<0$ and $-1<\lambda_{2}<-\frac{1}{10}$. Because $g(0)=-1<0, g\left(\frac{-1}{10}\right)=10-e>0$ and $g(-1)=1000-e^{10}<0$. Therefore $e^{\lambda_{1} t}$ and $e^{\lambda_{2} t}$ are two bounded solutions of (1.1) and convergent to 0 as $t \rightarrow+\infty$. In this paper, our motivation is the study of the asymptotic behavior of solutions to (1.1) 
in the case when $f$ and $p$ are nonconstant under the following suitable assumptions:

$$
\begin{gathered}
\int_{\tau}^{+\infty} t p(t) d t=+\infty \\
\int_{\tau}^{+\infty} t|f(t)| d t<+\infty \\
\limsup _{t \rightarrow+\infty} \int_{t-\tau}^{t} d s \int_{\tau}^{s} p(r) d r<1, \\
\lim _{t \rightarrow+\infty} \frac{f(t)}{p(t)}=0, \text { where } p(t)>0, \text { for sufficiently large } t>0 .
\end{gathered}
$$

Throughout the paper we assume that $p, f$, and $\phi$ are continuous. By a solution of equation (1.1), we mean a continuous function $u:[0,+\infty) \rightarrow \mathbb{R}$ which is twice continuously differentiable on $[\tau,+\infty)$ and satisfies the equation (1.1) for all $t \geq \tau$.

\section{MAIN RESULTS}

First, we prove two lemmas.

Lemma 1. Let $g: \mathbb{R} \rightarrow \mathbb{R}$ be continuously differentiable and bounded from above, then $\liminf _{t \rightarrow+\infty} g^{\prime}(t) \leq 0$.

Proof. Suppose to the contrary, $\liminf _{t \rightarrow+\infty} g^{\prime}(t)>\lambda>0$. Then there exists $t_{0}>0$ such that for each $t \geq t_{0}, g^{\prime}(t)>\lambda$. Integrating from $t_{0}$ to $T$, we have $g(T)-$ $g\left(t_{0}\right) \geq \lambda\left(T-t_{0}\right)$. We get a contradiction by letting $T \rightarrow+\infty$.

Lemma 2. Suppose that $g: \mathbb{R}^{+} \rightarrow \mathbb{R}$ is bounded from above and twice continuously differentiable such that

$$
g^{\prime \prime}(t) \geq-h(t), \quad \forall t \geq 0,
$$

where $h: \mathbb{R}^{+} \rightarrow \mathbb{R}^{+}$is continuous and $\int_{0}^{+\infty} t h(t) d t<+\infty$. Then there exists $\lim _{t \rightarrow+\infty} g(t)$ and $\limsup _{t \rightarrow+\infty} g^{\prime}(t) \leq 0$.

Proof. Integrating (2.1) from $t=S$ to $t=T$, where $S<T$, we get

$$
g^{\prime}(S) \leq g^{\prime}(T)+\int_{S}^{T} h(t) d t .
$$

Taking $\liminf$ as $T \rightarrow+\infty$, by Lemma 1 , we get

$$
g^{\prime}(S) \leq \int_{S}^{+\infty} h(t) d t
$$


Taking limsup as $S \rightarrow+\infty$, we derive that: $\lim \sup _{t \rightarrow+\infty} g^{\prime}(t) \leq 0$. Now, integrating (2.2) from $S=T_{1}$ to $S=T_{2}$, where $T_{1}<T_{2}$, and applying Fubini's theorem, we obtain

$$
\begin{gathered}
g\left(T_{2}\right) \leq g\left(T_{1}\right)+\int_{T_{1}}^{T_{2}} d S \int_{S}^{+\infty} h(t) d t \leq g\left(T_{1}\right)+\int_{T_{1}}^{+\infty} d S \int_{S}^{+\infty} h(t) d t \\
=g\left(T_{1}\right)+\int_{T_{1}}^{+\infty} d t \int_{T_{1}}^{t} h(t) d S=g\left(T_{1}\right)+\int_{T_{1}}^{+\infty}\left(t-T_{1}\right) h(t) d t \\
\leq g\left(T_{1}\right)+\int_{T_{1}}^{+\infty} t h(t) d t .
\end{gathered}
$$

Now, taking $\limsup$ as $T_{2} \rightarrow+\infty$ and $\liminf$ as $T_{1} \rightarrow+\infty$, the proof is complete.

Theorem 1. Suppose that $u$ is a solution to (1.1).

(1) If (1.2) and (1.3) are satisfied, then every eventually positive or eventually negative bounded solution to (1.1) converges to 0 as $t \rightarrow+\infty$.

(2) If (1.4) and (1.5) are satisfied, then every oscillatory solution to (1.1) converges to 0 as $t \rightarrow+\infty$.

Proof. (1) Assume that $u(t)$ is eventually positive and bounded solution of (1.1). The same proof works for eventually negative solution of (1.1). Then for large $t, u^{\prime \prime}(t) \geq f(t)=f^{+}(t)-f^{-}(t) \geq-f^{-}(t)$, where $f^{+}(t)=\max \{f(t), 0\}$ and $f^{-}(t)=\max \{-f(t), 0\}$. By Lemma 2 , there exists $\lim _{t \rightarrow+\infty} u(t)=l$ and $\limsup _{t \rightarrow+\infty} u^{\prime}(t) \leq 0$. Suppose that $l>0$, then there exists $t_{0}>\tau$ such that for each $t>t_{0}, u(t-\tau)>\frac{l}{2}$. Integrating (1.1) from $t>t_{0}$ to $T$, we get

$$
u^{\prime}(T)-u^{\prime}(t) \geq \frac{l}{2} \int_{t}^{T} p(s) d s-\int_{t}^{T} f^{-}(s) d s
$$

Taking $\lim \sup$ as $T \rightarrow+\infty$, we obtain

$$
-u^{\prime}(t) \geq \frac{l}{2} \int_{t}^{+\infty} p(s) d s-\int_{t}^{+\infty} f^{-}(s) d s, \text { for } t>t_{0} .
$$

If $\int_{\tau}^{\infty} p(s) d s=+\infty$, it is a contradiction; because $\int_{\tau}^{+\infty} f^{-}(t) d t<+\infty$. Otherwise, integrating from $t=t_{0}$ to $t=T$, we get

$$
-u(T)+u\left(t_{0}\right) \geq \frac{l}{2} \int_{t_{0}}^{T} d t \int_{t}^{+\infty} p(s) d s-\int_{t_{0}}^{T} d t \int_{t}^{+\infty} f^{-}(s) d s .
$$

Letting $T \rightarrow+\infty$, and by (1.2), (1.3) and Fubini's theorem, we get

$$
-l+u\left(t_{0}\right) \geq \frac{l}{2} \int_{t_{0}}^{+\infty} d t \int_{t}^{+\infty} p(s) d s-\int_{t_{0}}^{+\infty} d t \int_{t}^{+\infty} f^{-}(s) d s
$$




$$
=\frac{l}{2} \int_{t_{0}}^{+\infty}\left(t-t_{0}\right) p(t) d t-\int_{t_{0}}^{+\infty}\left(t-t_{0}\right) f^{-}(t) d t=+\infty .
$$

This is a contradiction. Then $l=0$.

(2) Suppose that $u(t)$ oscillates. Then, there exists a sequence $t_{n}$ of extreme points of $u(t)$ such that $t_{n} \rightarrow+\infty$ as $n \rightarrow+\infty$. Let $\mu>0$ such that

$\limsup \operatorname{sut}_{t \rightarrow+\infty} \int_{t-\tau}^{t} d s \int_{\tau}^{s} p(r) d r<\mu<1$ and let $0<\delta<\frac{1-\mu}{1+\mu}$. The sequence $\left\{t_{n}\right\}$ of extreme points of $u$, has a subsequence $s_{j}=t_{n_{j}}$ such that:

1) $u\left(s_{2 j}\right) \leq 0, u\left(s_{2 j+1}\right) \geq 0$, for all $j \geq 1$.

2) $s_{2 j}$ is a minimum point of $u$ in the interval $\left[s_{2 j-1}, s_{2 j+1}\right]$ and $s_{2 j+1}$ is a maximum point of $u$ in the interval $\left[s_{2 j}, s_{2 j+2}\right]$, for all $j \geq 1$.

It is enough to prove, $u\left(s_{j}\right) \rightarrow 0$ as $j \rightarrow+\infty$. By (1.1), we have

$$
\begin{array}{r}
u^{\prime \prime}\left(s_{2 j}\right)=p\left(s_{2 j}\right) u\left(s_{2 j}-\tau\right)+f\left(s_{2 j}\right) \Rightarrow u\left(s_{2 j}-\tau\right) \geq \frac{-f\left(s_{2 j}\right)}{p\left(s_{2 j}\right)} \\
u^{\prime \prime}\left(s_{2 j+1}\right)=p\left(s_{2 j+1}\right) u\left(s_{2 j+1}-\tau\right)+f\left(s_{2 j+1}\right) \\
\Rightarrow u\left(s_{2 j+1}-\tau\right) \leq \frac{-f\left(s_{2 j+1}\right)}{p\left(s_{2 j+1}\right)} .
\end{array}
$$

Give a subsequence $s_{j_{i}}$ of $s_{j}$ such that

$$
\begin{aligned}
s_{j_{1}}>\tau, s_{j_{i+1}}-s_{j_{i}}>\tau & \frac{|f(t)|}{p(t)}<\delta^{i}, \text { for each } t \geq s_{j_{i}}, \\
& \text { and } \int_{s_{n}-\tau}^{s_{n}} d s \int_{\tau}^{s} p(r) d r<\mu<1, \text { for each } n \geq j_{1} .
\end{aligned}
$$

Integrating (1.1) on $\left[s_{j_{i}}, s\right]$, we get

$$
u^{\prime}(s)=u^{\prime}(s)-u^{\prime}\left(s_{j_{i}}\right)=\int_{s_{j_{i}}}^{s} p(r) u(r-\tau) d r+\int_{s_{j_{i}}}^{s} f(r) d r .
$$

For large $m$ such that $s_{2 m}-\tau>s_{j_{i}}$, integrating (2.6) on [ $\left.s_{2 m}-\tau, s_{2 m}\right]$, and by (2.3), we obtain

$$
\begin{aligned}
u\left(s_{2 m}\right)+\frac{f\left(s_{2 m}\right)}{p\left(s_{2 m}\right)} \geq & u\left(s_{2 m}\right)-u\left(s_{2 m}-\tau\right)= \\
& \int_{s_{2 m}-\tau}^{s_{2 m}} d s \int_{s_{j_{i}}}^{s} p(r) u(r-\tau) d r+\int_{s_{2 m}-\tau}^{s_{2 m}} d s \int_{s_{j_{i}}}^{s} f(r) d r .
\end{aligned}
$$

Integrating (2.6) on $\left[s_{2 m+1}-\tau, s_{2 m+1}\right]$, and by (2.4), we get

$$
u\left(s_{2 m+1}\right)+\frac{f\left(s_{2 m+1}\right)}{p\left(s_{2 m+1}\right)} \leq u\left(s_{2 m+1}\right)-u\left(s_{2 m+1}-\tau\right)=
$$




$$
\int_{s_{2 m+1}-\tau}^{s_{2 m+1}} d s \int_{s_{j_{i}}}^{s} p(r) u(r-\tau) d r+\int_{s_{2 m+1}-\tau}^{s_{2 m+1}} d s \int_{s_{j_{i}}}^{s} f(r) d r .
$$

(2.7) and (2.8) imply that for sufficiently large $n$ such that $s_{n}-\tau \geq s_{j_{i}}$

$$
\left|u\left(s_{n}\right)\right| \leq \int_{s_{n}-\tau}^{s_{n}} d s \int_{s_{j_{i}}}^{s} p(r)|u(r-\tau)| d r+\int_{s_{n}-\tau}^{s_{n}} d s \int_{s_{j_{i}}}^{s}|f(r)| d r+\frac{\left|f\left(s_{n}\right)\right|}{p\left(s_{n}\right)} .
$$

By (2.5) for each $n \geq j_{i}$, we have

$$
\int_{s_{n}-\tau}^{s_{n}} d s \int_{s_{j_{i}}}^{s} p(r) d r<\mu
$$

and

$$
\int_{s_{n}-\tau}^{s_{n}} d s \int_{s_{j_{i}}}^{s}|f(r)| d r+\frac{\left|f\left(s_{n}\right)\right|}{p\left(s_{n}\right)}<\mu \delta^{i}+\delta^{i} .
$$

Consider $\mu+\delta^{i}+\mu \delta^{i}<\mu+\delta+\mu \delta<1$. From (2.9), we deduce that

$$
\left|u\left(s_{j_{2}}\right)\right| \leq M \mu+\delta+\mu \delta \leq(M+1)(\mu+\delta+\mu \delta),
$$

where $M=\max _{0 \leq n \leq j_{2}}\left\{\left|u\left(s_{n}\right)\right|\right\}$. Suppose that for $j_{2} \leq n \leq m$, we have

$$
\left|u\left(s_{n}\right)\right| \leq(M+1)(\mu+\delta+\mu \delta),
$$

then

$$
\begin{gathered}
\left|u\left(s_{m+1}\right)\right| \\
\leq \int_{s_{m+1}-\tau}^{s_{m+1}} d s \int_{s_{j_{1}}}^{s} p(r)|u(r-\tau)| d r+\int_{s_{m+1}-\tau}^{s_{m+1}} d s \int_{s_{j_{1}}}^{s}|f(r)| d r+\frac{\left|f\left(s_{m+1}\right)\right|}{p\left(s_{m+1}\right)} \\
\leq \max \left\{(M+1),\left|u\left(s_{m+1}\right)\right|\right\} \mu+\delta+\mu \delta .
\end{gathered}
$$

If $\left|u\left(s_{m+1}\right)\right|>M+1$, then

$$
\left|u\left(s_{m+1}\right)\right| \leq\left|u\left(s_{m+1}\right)\right| \mu+\delta+\mu \delta .
$$

Therefore

$$
\left|u\left(s_{m+1}\right)\right| \leq \frac{\delta+\mu \delta}{1-\mu} .
$$

This follows that $1>\frac{\delta+\mu \delta}{1-\mu}>M+1$. This is a contradiction. Therefore $\left|u\left(s_{m+1}\right)\right| \leq$ $M+1$. This implies that

$$
\left|u\left(s_{m+1}\right)\right| \leq(M+1) \mu+\delta+\mu \delta \leq(M+1)(\mu+\delta+\mu \delta) .
$$

Therefore, for all $n \geq j_{2}$, we get

$$
\left|u\left(s_{n}\right)\right| \leq(M+1)(\mu+\delta+\mu \delta) .
$$

Now, by induction suppose that

$$
\left|u\left(s_{n}\right)\right| \leq(\mu+\delta+\mu \delta)^{k}(M+1), \quad \text { for } n \geq j_{2 k} .
$$


By (2.9) for $n \geq j_{2 k+2}$, we get

$$
\left|u\left(s_{n}\right)\right| \leq \int_{s_{n}-\tau}^{s_{n}} d s \int_{s_{j_{2 k+1}}}^{s} p(r)|u(r-\tau)| d r+\int_{s_{n}-\tau}^{s_{n}} d s \int_{s_{j_{2 k+1}}}^{s}|f(r)| d r+\frac{\left|f\left(s_{n}\right)\right|}{p\left(s_{n}\right)} .
$$

If $r \geq s_{j_{2 k+1}}$, then $r-\tau \geq s_{j_{2 k+1}}-\tau \geq s_{j_{2 k}}$. By the hypothesis of induction and choosing the sequence $s_{n}$, we get

$$
|u(r-\tau)| \leq(M+1)(\mu+\delta+\mu \delta)^{k} .
$$

Now (2.11) implies that

$$
\begin{aligned}
& \left|u\left(s_{n}\right)\right| \leq(M+1)(\mu+\delta+\mu \delta)^{k} \mu+\delta^{2 k+1}+\mu \delta^{2 k+1} \\
\leq & (M+1)(\mu+\delta+\mu \delta)^{k} \mu+\delta^{k+1}+\mu \delta^{k+1} \leq(M+1)(\mu+\delta+\mu \delta)^{k+1},
\end{aligned}
$$

for $n \geq j_{2 k+2}$. This prove (2.10). The theorem is proved by letting $k \rightarrow+\infty$ in (2.10).

Corollary 1. If the conditions (1.2), (1.3), (1.4) and (1.5) are satisfied, then every bounded solution to (1.1) converges to 0 as $t \rightarrow+\infty$.

Now, we give some examples.

Example 1. Give $\tau=1, \phi(t)=\frac{1}{t+1}, p(t)=\left\{\begin{array}{l}\frac{1}{t^{2}-1}, \quad t>1 \\ 1, \quad t=1,\end{array}\right.$

and $f(t)=\frac{t^{2}-4 t-1}{t(t-1)(t+1)^{3}}$. The assumptions of Theorem 2.3 are satisfied, then every bounded and eventually positive (eventually negative) solution of (1.1) converges to 0 as $t \rightarrow+\infty$. As well as every its oscillatory solution converges to 0 . For example, $u(t)=\frac{1}{t+1}$ is a bounded solution of (1.1) with the above conditions which converges to 0 .

Example 2. $u(t) \equiv 1$ is a bounded and positive solution of

$$
\left\{\begin{array}{l}
u^{\prime \prime}(t)=p(t) u(t-\tau)-p(t), \quad t \geq \tau \\
u(t)=1, \quad 0 \leq t \leq \tau
\end{array}\right.
$$

where $p:[\tau,+\infty) \rightarrow \mathbb{R}^{+}$. But neither [(1.2) and (1.3)] nor (1.5) are satisfied and $u(t)$ is not convergent to 0 .

Example 3. $u(t)=$ sint is an oscillatory solution of

$$
\left\{\begin{array}{l}
u^{\prime \prime}(t)=u(t-\pi), \quad t \geq \pi \\
u(t)=\sin t, \quad 0 \leq t \leq \pi
\end{array}\right.
$$

But (1.4) are not satisfied and $u(t)$ is not convergent to 0 . 


\section{ACKNOWLEDGEMENT}

The author would like to thank the referee for his (her) careful reading of the paper and for the valuable comments on the article's corrections.

\section{REFERENCES}

[1] K. Gopalsamy, Stability and oscillations in delay differential equations of population dynamics, ser. Mathematics and its Applications. Dordrecht: Kluwer Academic Publishers, 1992, vol. 74.

[2] J. R. Graef and C. Qian, "Global attractivity in differential equations with variable delays," J. Aust. Math. Soc., Ser. B, vol. 41, no. 4, pp. 568-579, 2000.

[3] J. R. Graef, C. Qian, and B. Zhang, "Asymptotic behavior of solutions of differential equations with variable delays," Proc. Lond. Math. Soc. (3), vol. 81, no. 1, pp. 72-92, 2000.

[4] Y. Liu and W. Ge, "Asymptotic behavior of certain delay differential equations with forcing term," J. Math. Anal. Appl., vol. 280, no. 2, pp. 350-363, 2003.

[5] C. Qian and Y. Sun, "Global attractivity of solutions of nonlinear delay differential equations with a forcing term," Nonlinear Anal., Theory Methods Appl., Ser. A, Theory Methods, vol. 66, no. 3, pp. 689-703, 2007.

\section{Author's address}

\section{H. Khatibzadeh}

University of Zanjan, Department of Mathematics, Zanjan, Iran

E-mail address: hkhatibzadeheznu.ac.ir 